\title{
Low Dose Mapping of Semicrystallinity in Polymer Membranes with cryogenic 4D- STEM
}

\author{
Danielle Markovich ${ }^{1}$, Yue Yu ${ }^{1}$, Michael Colletta ${ }^{1}$, Megan Treichel ${ }^{2}$, Ryan Selhorst ${ }^{2}$, Cheyenne Peltier ${ }^{3}$, \\ Geoffrey Coates $^{3}$, Kevin J. Noonan ${ }^{2}$ and Lena Kourkoutis ${ }^{1}$
}

${ }^{1}$ School of Applied and Engineering Physics, Cornell University, United States, ${ }^{2}$ Department of Chemistry, Carnegie Mellon University, United States, ${ }^{3}$ Department of Chemistry and Chemical Biology, Baker Laboratory, Cornell University, United States

The understanding of the internal organization of soft materials such as polymer membranes is crucial in the development of new energy materials such as fuel cell membranes. TEM studies have played a major role in the characterization of the microphase separated domains of these materials and demonstrate their effects on their conductivities and mechanical properties [1,2]. The role of crystalline domains in these systems on ion conductivity, water uptake and durability presents another area of current interest [3]. Previous work has demonstrated the use of cryogenic scanning nanobeam electron diffraction (cryo-NBED) to visualize crystallinity in soft materials including polymer thin films with a resolution down to $10 \mathrm{~nm}$ [4]. Here, we show that reducing the dose by a factor of 10 enables imaging of small crystalline domains, just a few nanometers in size, within semicrystalline polymer membranes.

In a model system of hydrogenated polynorbornene $(\mathrm{hPN})$, we investigate the effect of incorporation of cationic groups, necessary for fuel cell applications, on crystallinity of the polymer membrane. Bulk measurements of the 5:1 hPN-co-iPrMe copolymer have shown a severe suppression of the membrane crystallinity due to incorporation of the cationic groups (iPrMe) [5]. The low degree of crystallinity in the copolymer (5-10\% compared to the hPN homopolymer) coupled with its radiation sensitivity presents a challenge in real-space imaging.

Figure 1 shows the results of cryo-NBED experiments performed with an electron microscope pixel array detector (EMPAD) on the copolymer and homopolymer at a dose of $\sim 7 \mathrm{e} / \AA^{2}$ and $\sim 3 \mathrm{e} / \AA^{2}$, respectively. The cryogenic 4DSTEM data were collected at $300 \mathrm{kV}$ on an FEI Titan Themis with a $3 \mathrm{~ms}$ acquisition time per pixel. The semiconvergence angle used was $0.57 \mathrm{mrad}$ resulting in a probe size of $\sim 2 \mathrm{~nm}$ and the pixel size was set to match the size of the probe. Cryogenic sample cooling as well as the use of a low electron dose enabled imaging of even the small $(\sim 5 \mathrm{~nm})$ domains in the copolymer (Fig. 1A). Py4DSTEM was used to analyze the raw data and detect the location of Bragg disks corresponding to crystalline domains in the specimen [6]. The domains are characterized by the orientation of the Bragg disks detected in each diffraction pattern. Examples of crystalline domain diffraction patterns are illustrated in Fig. 1A.i-ii. where we have summed nine convergent beam electron diffraction (CBED) patterns to more clearly show the weak Bragg disks in crystalline regions as well as the lack thereof in the amorphous regions (Fig. 1A.iii.).

A dose assessment is an essential part of the analysis of these soft materials. As a reference, the highly crystalline hPN homopolymer was used to determine a critical dose at which the crystalline domains are destroyed (Fig. 2) $[4,7]$. The same field of view was exposed and re-exposed multiple times using a dose of $\sim 3 \mathrm{e} / \AA^{2}$. As the total dose increases, a clear loss of crystallinity is observed demonstrating the necessity of low dose imaging for semicrystalline polymer materials. For the hPN homopolymer, even a dose of more than $\sim 10 \mathrm{e} / \AA^{2}$ results in severe damage which is also clearly visible in the virtual ADF images (Fig. 2). Coupling cryogenic cooling with low dose NBED imaging using a sensitive detector allows characterization of semicrystalline polymers with few-nanometer resolution.

This work was supported as part of the Center for Alkaline-based Energy Solutions (CABES), an Energy Frontier Research Center funded by the U.S. Department of Energy, Office of Science, Basic Energy Sciences under Award \# DE-SC0019445. 

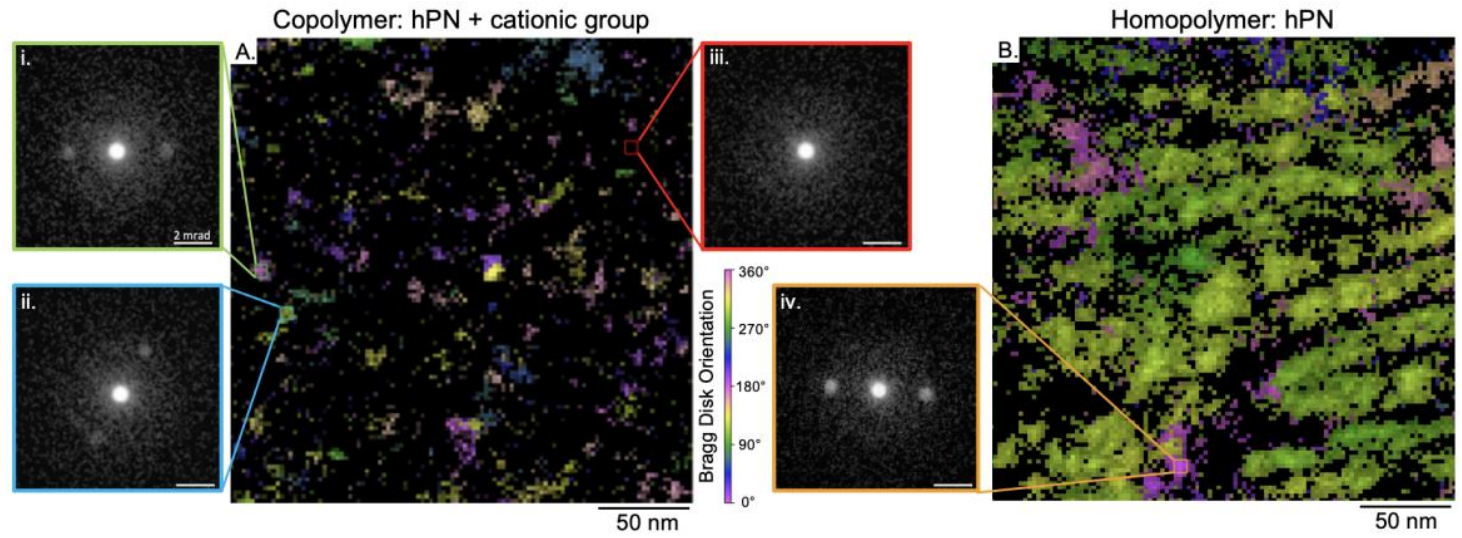

Figure 1. A-B) Cryogenic scanning nanobeam electron diffraction performed under low dose conditions $(\sim 7$ $\mathrm{e} / \AA 2$ and $\sim 3 \mathrm{e} / \AA 2$, respectively) enables mapping of crystalline domains in hPN-based polymer membranes. A) Incorporation of iPrMe cationic groups in a functionalized hPN copolymer strongly suppresses crystallinity compared to the hPN homopolymer (B). The domains are visually represented by identification of the orientation of the brightest Bragg disk in the diffraction pattern. Examples of CBED patterns from different domains are shown in $\mathrm{i}$, ii, and iv while the CBED pattern from a region with no crystalline detection is shown in iii. B) The hPN homopolymer shows higher crystallinity compared to the copolymer with larger domains and stronger Bragg disks observed in individual domains.

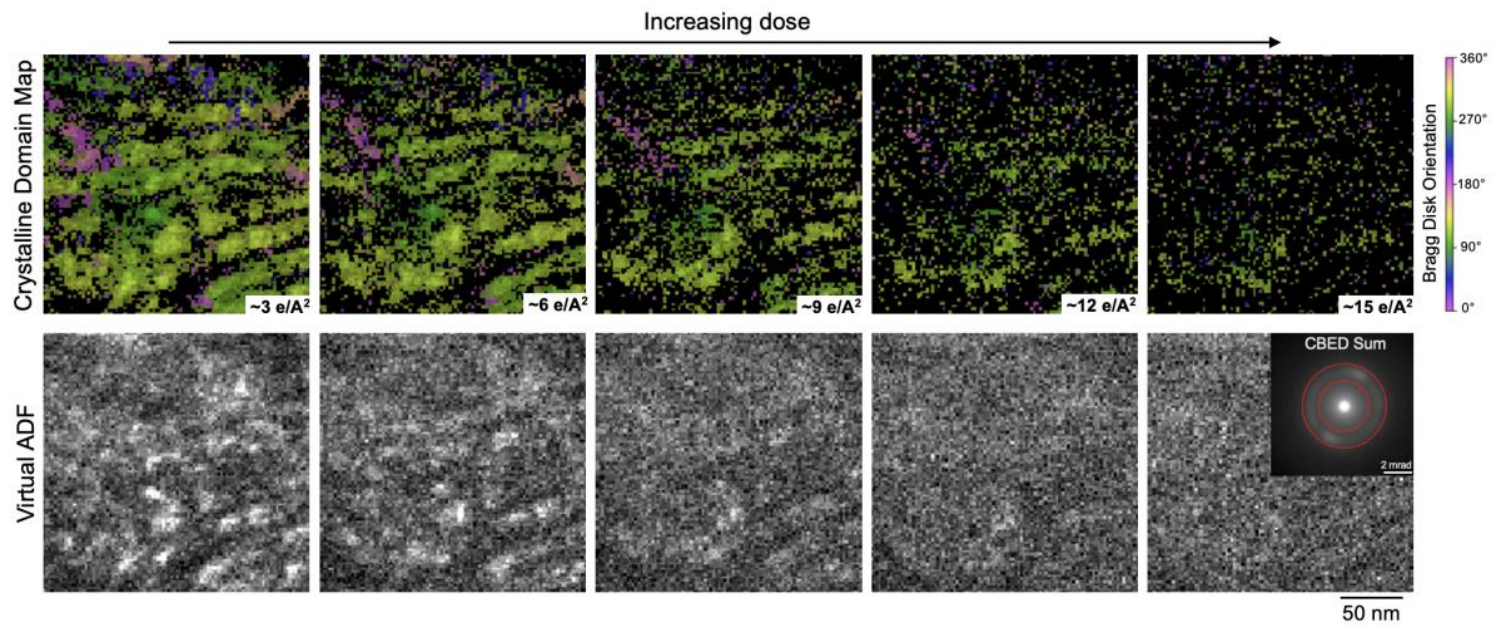

Figure 2. A dose series recorded on hPN over the same field of view demonstrates the destruction of crystalline domains even at a low dose of $\sim 10$ e/Å2. This loss in order is also clearly observed in the virtual ADF images (bottom row) obtained by integrating over an annulus corresponding to the location of crystalline Bragg disks as illustrated in the CBED sum.

\section{References}

[1] W. You, et al. Proceedings of the National Academy of Sciences 116, 9729-9734 (2019).

[2] F. I. Allen, et al. ACS Macro Letters 4, 1-5 (2015).

[3] N. C. Buggy, et al. ACS Applied Polymer Materials 2, 1294-1303 (2020).

[4] O. Panova, et al. Nature Materials 18, 860-865 (2019).

[5] M. Treichel, et al. Macromolecules 53, 8509-8518 (2020).

[6] B. H. Savitzky, et al. arXiv:2003.09523 (2020).

[7] L. W. Lee, R. A. Register. Macromolecules 38, 1216-1222 (2005). 